\title{
Spectroscopical Analysis of Strained Silicon Quantum Wells
}

\author{
J. McCarthy $^{* 1}$, E. Ni Mhuircheartaigh ${ }^{2}$, K. Lyutovich $^{3}$, M. Oehme ${ }^{3}$, T.S. Perova ${ }^{* * 1}$, R.A. Moore ${ }^{1}$, \\ W. Blau ${ }^{2}$
}

${ }^{1}$ Department of Electronic and Electrical Engineering, University of Dublin, Trinity College, Dublin 2, Ireland, ${ }^{2}$ Department of Physics University of Dublin, Trinity College, Dublin $2,{ }^{3}$ Ireland; Institut fuer Halbleitertechnik, Universitaet Stuttgart, Pfaffenwaldring 47, 70569 Stuttgart, Germany,

\begin{abstract}
In this study, Strained silicon Quantum Wells (QW) were characterised using a variety of micro-scopical techniques. Among the techniques used were Transmission Electron Microscopy (TEM), Elemental Electron Loss Spectroscopy (EELS), and micro-Raman spectroscopy. A combination of these methods facilitates investigation of the structure, the strain, and the dislocations present in such materials. Both conventional and High Resolution Transmission Electron Microscopy (HRTEM) are used to analyse strained silicon quantum wells (QW). These techniques allow for structure analysis at the atomic level. Elemental Electron Loss Spectroscopy (EELS) is used in tandem with other analytical techniques in order to give a quantitative analysis of the structures. The presence of various layers is independently verified using EELS, while layer depth and concentration profiles are also established. Relaxation levels in the virtual substrate as well as the strain in Si quantum wells are calculated using Raman spectroscopy.
\end{abstract}

Keywords: Silicon Quantum Wells, TEM, EELS, Raman spectroscopy

\section{INTRODUCTION}

Strained $\mathrm{Si}$ on strain-relaxed $\mathrm{SiGe}$ heterostructures is one of the more promising candidates for expanding $\mathrm{Si}$ complementary metal-oxide-semiconductor performance due to mobility enhancement of both electrons and holes in the strained Si channel layer ${ }^{1}$. Specifically, the strained Si film has greater electron and hole mobilities, which translates into greater drive current capabilities for NMOS and PMOS transistors, respectively ${ }^{2}$.

With the flexibility offered by bandgap and strain engineering, room temperature carrier mobility has been increased by a factor of two over the theoretical maximum for relaxed intrinsic bulk silicon ${ }^{3}$, and low temperature electron mobility has increased by a factor of ten over the best MOSFETs reported ${ }^{4}$. Electron mobility in a tensile-strained silicon well is improved over the performance of a silicon MOSFET by the reduced electron scattering from ionized impurities and surface roughness ${ }^{5}$ as well as reductions in intervalley scattering ${ }^{6}$. The enhanced carrier mobility in tensile-strained Si channel layers allows the development of high performance metal-oxide-semiconductor field-effect-transistors. The enhanced performances with tensile-strained Si layers are associated with the better electron confinement at the heterointerface between $\mathrm{Si}$ and $\mathrm{SiGe}$ and to the splitting of the degeneracy of the conduction valleys. These structures have been developed by MBE ${ }^{7,9,10}$ and UHV-CVD systems ${ }^{4,8}$. This paper investigates strained silicon quantum wells (QW) on top of thin, abrupt virtual substrates. Thin virtual substrates are considered as important for monolithic integration.

*jmccart2@tcd.ie; **perovat@tcd.ie; http://www.mee.tcd.ie; phone 3531 608-1432; fax 3531 677-2442; 
The virtual substrate consists of a silicon substrate and a strain-relaxed SiGe buffer (SRB). Strain relaxation is obtained by misfit dislocations (MD) at the interface. Important parameters are high degree of relaxation and low density of threading dislocations (TD).

Cross-sectional transmission electron microscopy (TEM) is used to investigate TD densities through the layers, with high resolution (HRTEM) focusing on inter-diffusion between the growth planes. Using analytical techniques, such as Elemental Electron Loss Spectroscopy (EELS), the structure of the material is verified and the widths and constituent elements are measured quantitatively. In addition, micro-Raman spectroscopy ${ }^{8}$, a non-destructive technique suitable to the calculation of strain and composition, is used to analyse the samples.

\section{SAMPLE STRUCTURE AND SAMPLE PREPARATION}

From a variety of samples, two with very different properties were selected to demonstrate the broad analytic range. Both samples had a rather high Ge content (40\%) to cover stress values up to $6 \mathrm{GPa}$. Sample C2971 was suspected of a having a medium degree of relaxation, while the other sample, C2972, was investigated for a high level of tensile strain in the $\mathrm{Si}$ well, but at the cost of a complicated threading dislocation structure.

The samples, C2971 and C2972, under investigation are shown in Fig.1 and Fig.2, respectively. Figure1 shows the strained Si QW spaced between constant composition SiGe barriers. For technological reasons, the structure is capped by a thin Si layer (5nm). Sample C2972 shown in Figure 2 has a structure very similar to Fig. 1 except that a a 40nm low temperature virtual substrate was placed below the SiQW in order to achieve a very high tensile strain in the $\mathrm{Si}$ QW.

\begin{tabular}{|c|}
\hline Si Cap 5nm \\
\hline 20nm SiGe $(40 \% \mathrm{Ge})$ \\
\hline 9nm Strained $\mathrm{Si}$ \\
\hline 200nm SiGe $(40 \% \mathrm{Ge})$ \\
\hline Si substrate \\
\hline
\end{tabular}

Fig.1 Structure schematic for C2971.

\begin{tabular}{|c|}
\hline Si cap 5nm \\
\hline $20 \mathrm{~nm} \mathrm{SiGe}(40 \% \mathrm{Ge})$ \\
\hline 9nm strained Si QW \\
\hline $200 \mathrm{~nm} \mathrm{SiGe}(40 \% \mathrm{Ge})$ \\
\hline LTVS $160^{\circ} \mathrm{C}(15+25) \mathrm{nm}$ \\
\hline Si substrate \\
\hline
\end{tabular}

Fig.2 Structure schematic for C2972.

Molecular Beam Epitaxy (MBE) was used in the growth of two distinct silicon quantum wells. Sample C2972 has an extremely thin (40nm) low temperature virtual substrate LTVS, as detailed in Fig 2, while the other sample, C2971, contains a SiGe layer with no LTVS present, as shown in Fig 1. It must be noted that for sample C2972 the growth was interrupted, with the wafer being removed and cleaned again after the initial 200nm SiGe layer, with Ge composition of $40 \%$, having been deposited. This was done to investigate the transfer of virtual substrates to other processing groups. Both samples C2971 and C2972 were prepared for TEM analysis in the usual way, with dimpling and ion milling.

Physically, a Si QW nominally consists of three or more different planar layers on a silicon substrate in which the doping and/or the germanium content are varied. The top four thin 'active layers', as shown in Fig.2, are the undoped silicon capping layer, a $\mathrm{Si}_{\mathrm{x}} \mathrm{Ge}_{1-\mathrm{x}}$ supply layer (this layer is generally called the supply layer as a higher channel occupation and a related threshold voltage shift can be achieved by doping this layer), the strained silicon well and an underlying $\mathrm{Si}_{1-\mathrm{x}} \mathrm{Ge}_{\mathrm{x}}$ layer. The well itself is formed by the conduction band offset in tensile-strained silicon ${ }^{11}$, as compared with the $\mathrm{Si}_{1-\mathrm{x}} \mathrm{Ge}_{\mathrm{x}}$. This allows electrons to be collected and separated from their ionized dopant atoms. The 
tensile strain is produced by the virtual substrate present inn both samples, with sample C2971 utilising the underlying barrier layer as the virtual substrate.

\section{MEASUREMENTS}

Bright-Field TEM measurements were recorded on a high-resolution microscope (HREM), JEOL 4000FX, with an accelerating voltage of $400 \mathrm{KV}$. The angle of rotation and tilt were adjusted to give suitable images while a diffraction aperture was inserted for maximum contrast. EELS measurements were performed on a Zeiss Em912 Omega microscope with a Lanthanium hexaborid cathode source and an operating voltage of 120kV. The Energy loss spectra were recorded on a Gatan 1024 x 1024 slow scan CCD camera. Raman spectra were registered in backscattering geometry using a RENISHAW 1000 micro-Raman system equipped with a Leica microscope. To prevent sample heating the power density was kept below $10^{5} \mathrm{~W} / \mathrm{cm}^{2}$. The measurements were performed at room temperature with $514.5 \mathrm{~nm}$ line of an $\mathrm{Ar}^{+}$laser. An 1800 lines $/ \mathrm{mm}$ grating was used in all measurements, which corresponds to a spectral resolution of $\sim 2.5 \mathrm{~cm}^{-1}$ per 3 pixels. The 100 times magnifying objectives of the Leica microscope focuses the beam into spot of about 1 micrometer in diameter.

\section{Results and Discussion}

\subsection{TEM}

TEM investigation, using different magnifications and angle of tilt, were used to analyse both samples. Layer interfaces, misfit dislocations and threading dislocations were investigated throughout the structures. Fig.3 shows a bright field TEM image, showing clearly the distinct layers present. A low TD density is clearly present in the SiGe layers with MD at the $\mathrm{SiGe} / \mathrm{Si}$ substrate interface. Subsequent TEM images at a variety of tilt angles revealed similar conditions.

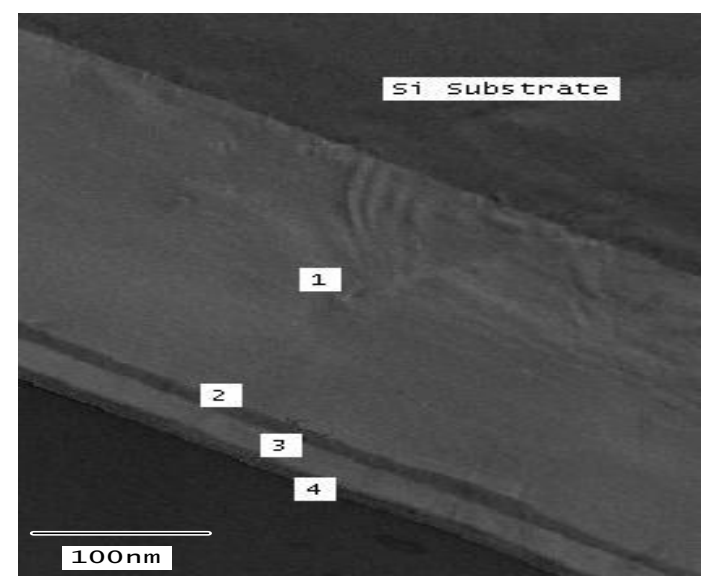

Fig. 3 Bright Field C2971 TEM image, with 5 distinct layers: Silicon Substrate, (1) 200nm SiGe layer, Ge 40\%, (2) 9nm Strained $\mathrm{Si},(3) 20 \mathrm{~nm} \mathrm{SiGe} \mathrm{(40 \% ),} \mathrm{(4)} 5 \mathrm{~nm}$ Si cap.

Though TDs are present in the SiGe layer, their density is low, with terminations either in the prepared TEM foil, as indicated in Fig.4 by TD1 or in the SiQW, as shown by TD2. Only dislocations running parallel to the prepared TEM foil (typically 100-400nm thick) are visible at their full length.

This TEM image of Fig.4 is in marked contrast to the Bright Field image for sample C2972 of Fig.5 which shows a high threading dislocation density running from the $\mathrm{Si} / \mathrm{SiGe}$ interface all through the material's layers. The contrast in TEM images is due to sample growth, with sample C2972 using a 40nm thick low temperature virtual substrate, while C2971 does not. In the case of C2972, the TD density is very high, moving from the interface of the Si bulk right across the layers. Various points are marked off along the micrograph of Fig.5 and can be explained as follows. In the growth of sample C2972, two MBE setups were used. After the very-low-temperature growth stage and with a total thickness of $200 \mathrm{~nm}$ in the initial MBE system, the wafer was unloaded and given an additional pre-epitaxial cleaning. The 
sample was then loaded into the second MBE setup, where a SiGe layer was overgrown with the final QW structure. Line 2 in this image is the boundary caused by this reloading. Line 1 is probably an accidental effect. The strained Si QW is at 3 while the cap is found at 4.

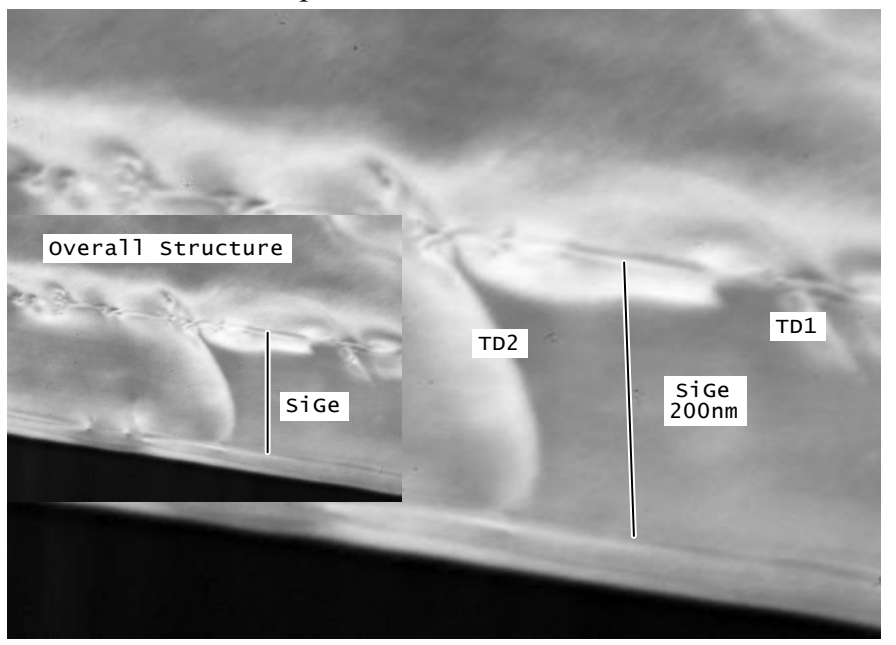

Fig. 4 Bright Field Image of C2971 focusing on TD, with overall image inserted also. Different TDs are seen running through the $\mathrm{SiGe}$ layer.

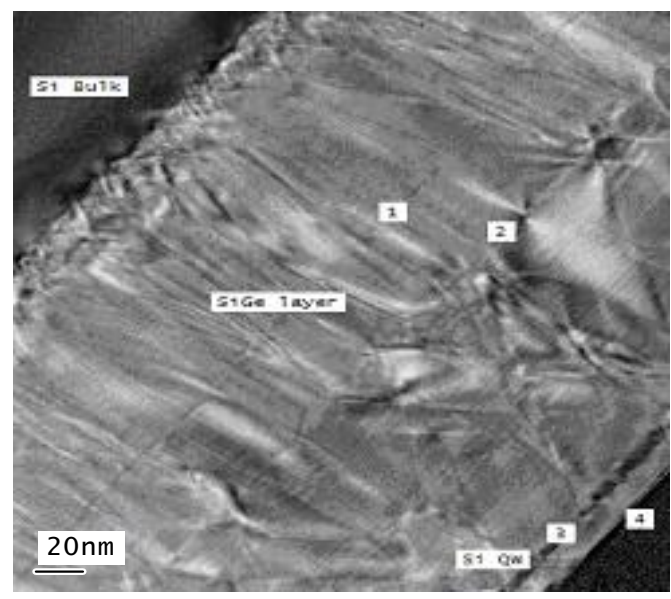

Fig.5. TEM image of C2972, showing clearly the high density of threading dislocations present in the material. TDs dominate the material layers due to the high stress caused by LTVS stage in wafer growth.

\subsection{EELS}

Using EELS mapping, independent of whether the Ge or Si edge is used, one can determine the compositional map of the material, and the percentage of materials present using ratios of counts (the TEM foil thickness should be constant). The disparity in counts moving from left to right in Fig.7 is indicative of the influence of the foil thickness on the measured data. A thickness per lambda profile was developed across the same area and corresponded to an increasing foil thickness when moving towards the bulk. Once this thickness per lambda is taken into account, percentage compositional values can be calculated and are found to correspond with expected values.

In Elemental Electron Loss Spectroscopy, both L and K edge filtering is possible for Si and Ge materials. Both were used initially to establish the most suitable operating environment for the samples under investigation. However, switching to the $\mathrm{K}$ edge $\mathrm{Si}$ excitation gives us a clearer picture that is less influenced by the topographical nature of our specimen. The $\mathrm{K}$ edge is at $1869 \mathrm{ev}$ while the window used was $60 \mathrm{eV}$. Similarly, for Ge, a $\mathrm{K}$ edge mapping was performed. 


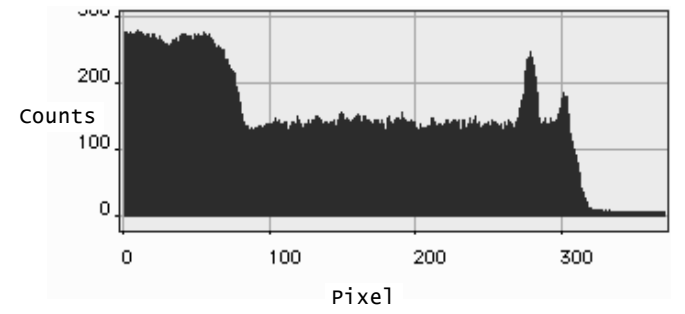
where Si content of $60 \%$ is present. The two peaks on the right hand side of Fig.7 correspond to the Si well and Si cap respectively. The valley between these peaks is indicative of the presence of the $20 \mathrm{~nm}$ SiGe layer with Ge composition of $\sim 40 \%$.

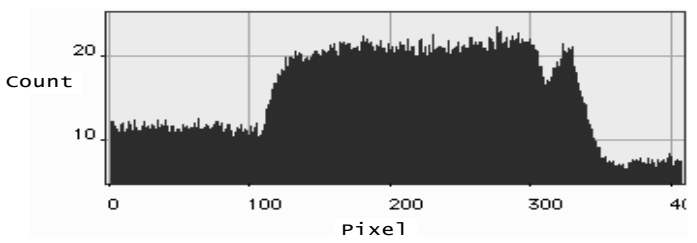

Fig.8. EELS Germanium Profile moving from bulk to cap.

Similarly for Ge compositional analysis, an EELS profile was developed, as shown in Fig.8. Fig.8 is a raw profile map, ignoring the influences of thickness but once again a clear pattern emerges. Moving from the bulk area, the Ge profile increases ( as expected) when in the SiGe layer, and falls off when the Si QW is encountered (at pixel $=300$ approx). The top SiGe layer is clearly present as indicated by the peak after the QW. Conversion of data from pixel into actual real dimensions is a straight forward process. The actual thickness of the layers is in good agreement with the nominal one. Again, this information is cross-checked and verified using HREM.

Similarly, for sample S2972 an EELS map was developed that allows us to determine the materials present, as well as indicating both size and compositional ratios. A K edge was used for both Si and Ge profile mapping as before and the results are interpreted below.Fig.9 shows a Si profile map of S2972, moving from the Si cap into the bulk, in contrast to the setup used for S2971.

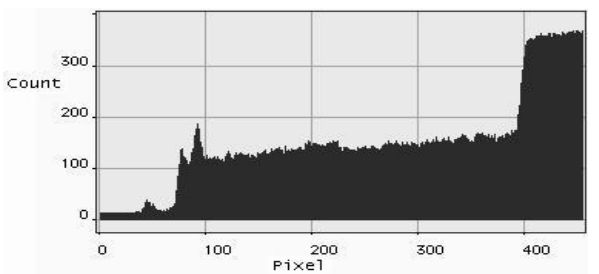

Fig.9. Si (K edge) Profile map for C2972.

Moving from left to right in Fig.9, the two distinct peaks indicating Si cap and well respectively, as well as the dominant feature of the bulk are noted. The composition of layers in between these peaks is determined using an EELS Ge profile map, shown in Fig. 10.

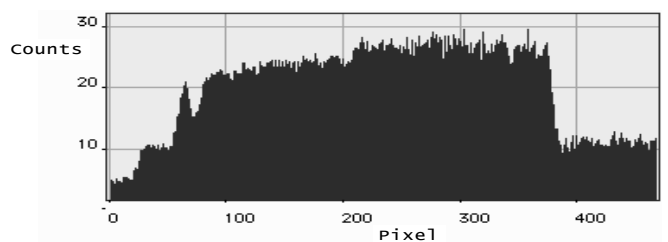

Fig.10 EELs Ge Map moving from cap to bulk.

Noise peaks and thickness influences are subsequently removed to give accurate data readings

The determination of sample composition is clearly evident from the EELs profile maps. 


\subsection{Raman spectroscopy}

The Raman spectrum of crystalline Si has a characteristic peak at $520 \mathrm{~cm}^{-1}$ which corresponds to longitudinal optical (LO) phonon vibrations of the Si-Si bond. For Ge, the reported Raman spectrum is seen at $300 \mathrm{~cm}^{-1}$. In SiGe layer Si-Si peak will be shifted to the low-frequency side, i.e. expected to be at around $500 \mathrm{~cm}^{-1}$, depending on Ge content (x) and stress in the layer. Other groups of phonon modes observed in the Raman spectrum of $450-400 \mathrm{~cm}^{-1}$ and $240-300 \mathrm{~cm}^{-1}$ belong to the Si-Ge and Ge-Ge stretching modes respectively. The presence of Si-Si peaks associated with Si substrate (or Si buffer layer), will depend on how deeply the laser probe beam will penetrate into the sample during Raman measurements. This will depend on the absorption coefficient $(\alpha)$ of the material at the wavelength of the exciting laser line and can be roughly assessed by using a simple expression ${ }^{12}$ for the depth of penetration $\left(d_{p}\right)$ for pure $\mathrm{Si}$ and $\mathrm{Ge}$

$$
d_{p}=2.3 / 2 \alpha
$$

and for SiGe layer (linear interpolation)

$$
d_{p}(\mathrm{SiGe})=(1-\mathrm{x}) d_{p}(\mathrm{Si})+\mathrm{x} d_{p}(\mathrm{Ge}) .
$$

Using $\alpha_{\mathrm{si}}=15080 \mathrm{~cm}^{-1}$ and $\alpha_{\mathrm{Ge}}=600000 \mathrm{~cm}^{-1}$ at $\lambda=514.5 \mathrm{~nm}$, we estimate $\mathrm{d}_{\mathrm{p}}(\mathrm{Si}) \approx 763 \mathrm{~nm}$ and $\mathrm{d}_{\mathrm{p}}(\mathrm{Ge}) \approx 19.2 \mathrm{~nm}$. From Eqn. (2) for $x=0.4$ we can estimate that the laser light will penetrate through the entire layers structure and only partly penetrate to the Si substrate for both samples under investigation. Raman spectra shown in Figs. $6 a$ and $b$, confirm this estimation, since in both spectra the peak at $520 \mathrm{~cm}^{-1}$ from the substrate is seen. The Si-Si peak at $520 \mathrm{~cm}^{-1}$, corresponding to the substrate, is more pronounced in sample C2971 due to the different structure of SiGe layer beneath Si QW layer as shown in Figs. 1 and 2. However, the intensity of this peak for sample C2972 is significantly smaller than can be expected from the presence of the additional $40 \mathrm{~nm}$ thick $\mathrm{Si}_{0.6} \mathrm{Ge}_{0.4}$ layer. We believe that this is due to the impact of high threading dislocations presented in C2972 which causes additional losses of light for the scattering.
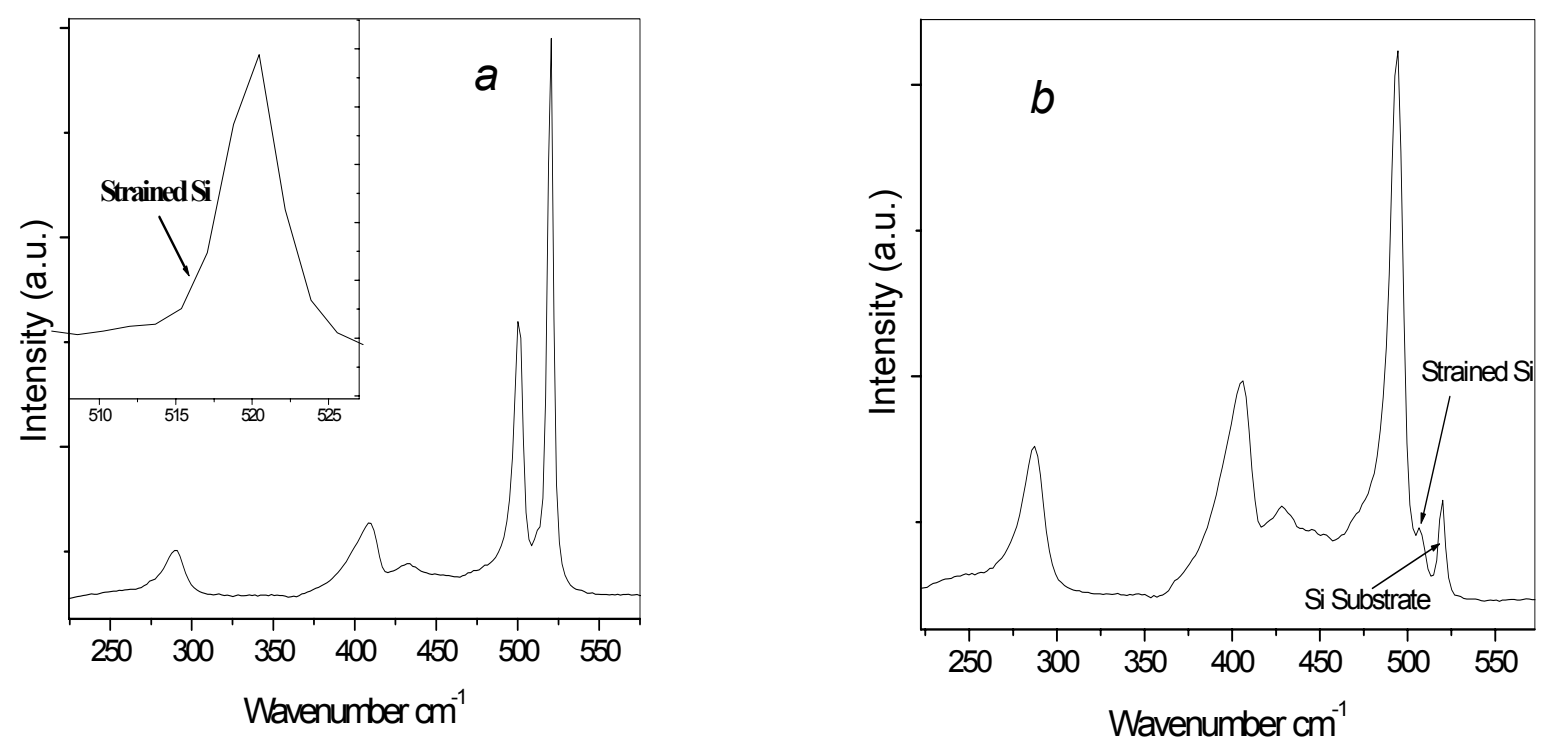

Fig.6 Raman spectra of sample (a) C2971 and (b) C2972.

Analysis of these sandwich-type structures using Raman spectroscopy is not so trivial due to reciprocal influence of neighbouring layers. Careful curve fitting and certain assumptions are necessary in order to extract the relevant information. In particular, for further analysis we assumed that the major contribution to the phonon lines from SiGe layer originates from $\mathrm{Si}_{0.6} \mathrm{Ge}_{0.4}$ layer beneath $\mathrm{Si} \mathrm{QW}$, since the thickness of this layer is much greater than the thickness 
of other SiGe layers in the structure. The same assumption has been made with regards to Si QW layer which is nearly twice as large as strained Si cap layer.

By measuring the experimental position of Si-Si $\left(\omega_{\mathrm{SS}}\right)$ and $\mathrm{Si}-\mathrm{Ge}\left(\omega_{\mathrm{SG}}\right)$ phonon lines for SiGe alloy, the Ge content $(x)$ and strain $(\varepsilon)$ in SiGe layer can be determined by combination of the following equations ${ }^{13-15}$.

$$
\begin{aligned}
& \omega_{\mathrm{SS}}(x)=520.0-68 \mathrm{x}-830 \varepsilon \\
& \omega_{\mathrm{SG}}(x)=400.5+12 \mathrm{x}-575 \varepsilon
\end{aligned}
$$

The resulting Ge concentration in $200 \mathrm{~nm}$ SiGe layer was found to be $\sim 40 \%$ for both samples, matching the expected growth parameters. The performed analysis shows essential difference in degree of relaxation, R, of SiGe layer for two samples. Sample C2972, which was fabricated with using a very-low-temperature SiGe buffer is much more relaxed with $\mathrm{R} \approx 90 \%$, while for $\mathrm{C} 2971$ the degree of relaxation is calculated at $\sim 49 \%$.

Stress, $\sigma$, in Si QW were estimated from the position of Si-Si phonon mode using the following expression ${ }^{16}$

$$
\sigma=\Delta \omega / 2 \times 10^{9} \mathrm{~Pa}
$$

while strain, $\varepsilon$, was determined by the following very good estimation ${ }^{17}$

$$
\begin{aligned}
& \qquad \varepsilon=\left|\Delta \omega_{S i, \exp }\right| /\left|\Delta \omega_{S i, \max }\right| \\
& \text { and } \quad\left|\Delta \omega_{S i, \max }\right|=|b| \cdot\left[a_{0}(x)-a_{0}\right] / a_{0} \\
& \text { with } \quad a_{0}(x)=0.5431+0.02 x+0.0026 x^{2}
\end{aligned}
$$

where $\Delta \omega_{\mathrm{Si} \text { exp }}$, is the measured Raman shift, $\mathrm{b}=930 \mathrm{~cm}^{-1}$ the strain-phonon coefficient and $a_{0}=0.5431 \mathrm{~nm}$ the lattice constant for unstrained Si.

The position of Si-Si peak for QW layer was obtained after the fitting of the complicated Raman spectra in the range 470-550 $\mathrm{cm}^{-1}$. The Si-Si peak for strained QW layer is seen very well in spectrum shown in Fig. 6b for samples C2972, whilst for samples C2971 this is not so pronounced and can only be seen as a shoulder on the $520 \mathrm{~cm}^{-1}$ line shown in the insert of Fig. 6a. After the fitting of both spectra the position of Si-Si peak for QW layer was found to be at $512 \mathrm{~cm}^{-1}$ for sample C2971 and at $508 \mathrm{~cm}^{-1}$ for C2972. These correspond to the tensile stress of $4 \mathrm{GPa}$ and $6 \mathrm{Gpa}$ respectively. The strain values are 55\% for C2971 and 83\% for C2972. The results obtained from Raman measurements are in a good correlation with TEM analysis.

\section{Conclusion}

Strained Silicon Quantum Wells were characterised vis-a-vis structure, strain, relaxation and dislocations using a variety of spectroscopic techniques, including TEM, EELS, and Raman. Both conventional TEM and HREM was used to analyse strained silicon quantum wells (QW) in relation to TDs. The contrast in TEM images is due to sample growth, with sample C2972 using a 40nm thick low temperature virtual substrate, while C2971 does not. In the case of C2972, the TD density is very high, moving from the interface of the Si bulk right across the layers. Using EELS, the elemental structure of the layers was verified, with a quantitative analysis of layer widths and constituent components obtained. The presence of various layers is independently verified using EELS, while layer depth and concentration profiles are also established. Relaxation levels in the virtual substrate as well as the strain in Si QW are calculated using Raman spectroscopy. It has been shown that the use of a LTVS can lead to a higher relaxed SiGe buffer layer and higher tensile strain in Si but in turn leads to an increase in the density of threading dislocations. 


\section{ACKNOWLEDGMENTS}

The authors would like to thank the Marie Curie Fellowship and the Max-Planck-Institut fuer Metallforschung Stuttgart for providing training and use of their facilities. The financial support towards this work from HEA Ireland (PRTLI Grant) is greatly acknowledged. Fruitful discussions with E. Kasper about virtual substrate concepts and analysis of SiGe layers are thankfully acknowledged.

\section{REFERENCES}

[1] A O'Neill G and D A Antoniadis 1996 IEEE Trans. Electron Devices 43911

[2] R People 1986 IEEE J. Quantum Electron. 22 1696-71

[3] Nelson S F, Ismail K, Chu J O and Meyerson B S 1993 Appl. Phys. Lett. 63367

[4] Ismail K, Arafa M, Saenger K L, Chu J O and Meyerson B S 1995 Appl. Phys. Lett. 661077

[5] Gold A 1987 Phys. Rev. B 35723

[6] Miyata H, Yamada T and Ferry D K 1993 Appl. Phys. Lett. 622661

[7] Fernandez J M, Matsumura A, Zhang X M, Xie M H, Hart L, Zhang J, Joyce B A and Thornton T J 1995 J. Mater. Sci.: Mater. Electron. 6330

[8] Legoues F K, Meyerson B S and Morar J F 1991 Phys. Rev. Lett. 662903

[9] Schäffler F 1997 Semicond.Sci. Technol. 121515

[10] Lyutovich K, Kasper E, Ernst F, Bauer M, Oehme M 2000 Mat. Sci. Eng. B71 14

[11] Kasper E, Lyutovich K 2004 Solid-State Electronics 481257

[12] European Commission 1992, Raman and Luminescence Spectroscopy for Microelectronics, Catalogue of optical and physical parameters "Nostradamus" project SMT4-CT-95-2024

[13] Alonso M.I, Winer K, 1989 Phys.Rev.B 39, 10056 - 10062

[14] B. Dietrich, E. Bugiel, J. Klatt, G. Lippert, T. Morgenstern, H.J. Osten, and P. Zaumseil, J. Appl. Phys., 74, 3177 (1993).

[15] J.C. Tsang, P.M. Mooney, F. Dacol, and J.O. Chu, J.Appl.Phys., 75, 8098 (1994).

[16] Th. Englert, G. Abstreiter, J. Pontchara, Solid-State Electron., 23, 31 (1980).

[17] R. Liu, Syt. Zolliner, M. Liaw, D. O’Meara and N. Cave, Mater.Res.Symp.Proc., 533, 63 (1998). 